\title{
377 CHARACTERIZATION OF PERIPHERAL BIOMARKERS OF GS-1423, A FIRST IN CLASS BIFUNCTIONAL ANTI-CD73- TGF $\beta$ RECEPTOR II- TRAP MOLECULE, IN A PHASE 1 DOSE ESCALATION STUDY IN PATIENTS WITH ADVANCED SOLID TUMORS
}

${ }^{1}$ Marianna Zavodovskaya* ${ }^{2}$ Anthony Tolcher, ${ }^{3}$ Michael Gordon, ${ }^{4}$ James Strauss, ${ }^{5}$ Kathleen Mahoney, ${ }^{1}$ Ping Cheng Yi, ${ }^{1}$ Rick Sorensen, ${ }^{6}$ Xiaoyun Yang, ${ }^{1}$ Kai-Wen Lin, ${ }^{1}$ Biao Li, ${ }^{1}$ Anna Seto, ${ }^{1}$ Matthew Peach, ${ }^{1}$ Audrey Goddard, ${ }^{1}$ Tianling Chen, ${ }^{1}$ Juliane Jürgensmeier. ${ }^{1}$ Gilead Sciences, Woodside, CA, USA; ${ }^{2} N E X T$ Oncology, San Antonio, TX, USA; ${ }^{3}$ HonorHealth Research Institute, Scottsdale, AZ, USA; ${ }^{4}$ Mary Crowley Research Center, Dallas, TX, USA; ${ }^{5}$ Beth Israel Deaconess Medical Center, Boston, MA, USA; ${ }^{6}$ Roche, South San Francisco, CA, USA

Background GS-1423 is a first-in-class bifunctional molecule comprised of an anti-CD73 antibody fused to the extracellular domain of TGF $\beta$ receptor II (TGF $\beta$ RII). GS-1423 is designed to inhibit CD73-mediated adenosine production and neutralize active TGF $\beta$ within the tumor microenvironment. Dual antagonism of these 2 broadly immunosuppressive barriers is anticipated to facilitate productive anti-tumor immunity.

Methods This open label Phase 1a study (NCT03954704) evaluated the safety, tolerability, and pharmacokinetics of GS1423. Exploratory biomarkers included the evaluation of the inhibition of GS-1423 targets, i.e.CD73 and TGF $\beta$, in the periphery. Biomarker assessments were performed in serial blood samples from patients receiving GS-1423 every two weeks (Q2W). Biomarker assays, unless otherwise stated, were custom built and qualified to measure the following: 1) TGFbeta $1 / 2 / 3$ (Luminex, Bio-Rad) in platelet poor plasma, 2) CD73 target occupancy (TO) on B and CD8 T cells in whole blood, 3) free soluble CD73 (sCD73) not bound to GS-1423, and 4) sCD73 activity in platelet poor plasma. Biomarker values were plotted longitudinally by patient and by dose.

Results A dose dependent decrease in TGF-beta $1 / 2 / 3$ in plasma of patients was observed on treatment. There was no detectable TGF $\beta$ at the $20 \mathrm{mg} / \mathrm{kg}$ dose level and above at 2 hours post first dose and for the duration of the Q2W dosing interval. A dose dependent increase in CD73 TO on B and CD8 T cells was also observed with treatment, and complete TO was achieved at $20 \mathrm{mg} / \mathrm{kg}$ and above at 2 hours post first dose for the duration of the Q2W dosing interval. Free sCD73 decreased at 2 hours post first dose, while remaining above the lower limit of quantitation, and then increased above baseline after 24 hours post-dose at the $3 \mathrm{mg} / \mathrm{kg}$ dose level and above. The sCD73 activity in blood correlated with changes in free sCD73 levels.

Conclusions Blood biomarker analyses of GS-1423 in patients with advanced solid tumors demonstrated undetectable soluble TGF $\beta 1 / 2 / 3$ and complete TO of CD73 on B and T cells at the $20 \mathrm{mg} / \mathrm{kg}$ dose level and above. The mechanism underlying the increase in sCD73 following GS-1423 treatment remains to be elucidated.

Ethics Approval The study obtained ethics approval from the IRB/IEC and all participants gave informed consent before taking part in the study.

http://dx.doi.org/10.1136/jitc-2021-SITC2021.377 